\title{
Title: Structural basis for antagonism of bacterial LPS transport
}

\author{
Presenting Author: Christopher M. Koth, Director of Structural Biology, Genentech
}

Additional Authors: Hoangdung Ho, Anh Miu, Mary Kate Alexander, Natalie K. Garcia, Angela Oh, Inna Zilberleyb, Mike Reichelt, Cary Austin, Christine Tam, Stephanie Shriver, Huiyong Hu, Sharada S. Labadie, Jun Liang, Lan Wang, Jian Wang, Yan Lu, Hans E. Purkey, John Quinn, Yvonne Franke, Kevin Clark, Maureen H. Beresini, Man-Wah Tan, Benjamin D. Sellers, James R. Kiefer, Till Maurer, Aaron T. Wecksler, Michael Koehler, Vishal Verma, Yiming Xu, Mireille Nishiyama, Jian Payandeh

Affiliation: Genentech Inc., Department of Structural Biology, South San Francisco, CA, USA 94080; email: kothc@gene.com

\begin{abstract}
High-resolution structural data has provided novel insights into the molecular basis of lipid flipping by $A B C$ transporters, however complementary information on mechanisms of antagonism has been lacking due to significant challenges in the discovery of selective modulators. MsbA is an essential bacterial ABC transporter that flips lipopolysaccharide (LPS) across the inner membrane of Gram-negative bacteria. We have discovered potent and selective inhibitors of MsbA with antibacterial activity and determined crystal structures of the transporter in complex with these small molecule antagonists. The structures reveal that the inhibitors trap MsbA an inward-facing and substrate-bound conformation, thereby preventing the transition to an outward-facing state. In addition, inhibitor binding is allosterically coupled to a second mode of antagonism $\sim 60 \AA$ away from the transmembrane binding site, where the nucleotide binding domains are found in an asymmetric conformation, catalytically and structurally uncoupled from the transporter. Our study further elucidates the mechanisms underlying the selective recognition and transport of a complex membrane lipid and establishes a structural template for the design of new antibiotics and modulators of other $A B C$ transporters.
\end{abstract}

